\title{
Is Neuroscience Compatible with a Belief in God? A Perspective from the Thalamus
}

\author{
Zoran Vukadinovic ${ }^{1, *}$ \\ ${ }^{1}$ Addiction Research and Treatment Services, University of Colorado School of Medicine, Denver CO \\ "Corresponding author: Zoran Vukadinovic, Addiction Research and Treatment Services, University of Colorado School of Medicine, Denver CO. E-mail: zvukadi@gmail.com
}

Received 2017 June 27; Accepted 2017 July 22.

\begin{abstract}
Microarchitecture of thalamic inputs suggests that the main information that reaches the cortex trans-thalamically contains copies of motor instructions issued to lower motor centers in the central nervous system. This includes all sensory inputs that reach the cortex with the sole exception of olfaction. Moreover, all cortical areas, regardless of their classification as sensory, motor or associational, contain layer VB neurons that have branching axons that innervate both lower motor centers in the CNS and thalamic relays. These findings together challenge the sensory versus motor dichotomy within the thalamus and the cortex and suggest that all thalamocortical interactions are to some extent both, sensory and motor. This means that agency and perception are inseparable. All experience is, therefore, necessarily a model for an agent. An important feature of all models is that they have outside requirements to function as such. They are not ends in themselves. The necessary background for a model can be provided by other more basic models, which is something that contemporary science has done very successfully. However, science, which is itself a model, ultimately requires the existence of something that is unmodelable. Therefore, being a scientist and believing in God is not contradictory.
\end{abstract}

Keywords: Thalamus, Perception, Efference Copies, Modelability, Theology

\section{Introduction}

The brain may be the most complex structure in the known universe, while the issue of God's existence is one of the most profound questions that humanity has faced. Therefore, attempting to synthesize the two questions is both important and difficult. As we are learning more and more about the human nervous system and how it generates our experience of reality, some are certainly asking ourselves what this can tell us about the existence of God and the divine nature?

Historically, neuroscience has been used as evidence that God does not exist. If we can explain our thoughts and feelings in materialistic neurobiological terms, then, as this logic goes, we do not need concepts such as God and the soul. Here, I will attempt to provide an alternative account, to demonstrate that a belief in one God is compatible with what neuroscience is teaching us. In this essay, we will focus on thalamocortical interactions, which are important because they generate our subjective experience (1).

\section{The Partnership Between the Thalamus and the Cor- tex}

The thalamus and the cortex are extensively interconnected and the partnership between them underlies our conscious experience; there is no consciousness without it. The thalamus has been identified as a major target of most anesthetic agents (2). Additionally, localized thalamic strokes are associated with persistent loss of consciousness (3). All sensory information that reaches the cortex does so trans-thalamically. The only exception here is olfaction. Thus, thalamocortical interactions are important to describe in any exploration of how conscious experience, including perception, arises.

The inputs to the thalamus can be subdivided into two categories, driver and modulator $(4,5)$. Broadly speaking, the former transmit information while the latter modulate how that information is transmitted. They are distinguished based on morphological, neuroanatomical and neurophysiological criteria. We will focus here on the driver inputs. They have thicker axons, larger and more proximal terminals; they utilize ionotropic glutamatergic receptors only and produce larger excitatory post-synaptic potentials. All of these features are consistent with their role as the main information transmitting inputs.

The neurons that provide driver inputs to the thalamus, regardless of their source, branch and innervate lower motor centers in the central nervous system. This suggests that all information that arrives in the thalamus for relay to the cortex contains copies of motor commands to those motor centers. To use an example from the visual 
system, axons of the optic tract, on their way from the optic chiasm to the thalamus, branch and 1, provide driver inputs to the lateral geniculate nucleus (LGN) and 2, innervate the superior colliculus and the pretectal nucleus in the midbrain, which are motor centers that control eye movements and the pupillary response, respectively. To use another example, again from vision, the primary visual cortex, which receives visual information from the LGN, contains layer VB neurons that likewise branch and provide 1 , driver inputs to the thalamus and 2 , innervation to the superior colliculus and the pretectal nucleus. We could give similar examples from other sensory modalities with the exception of olfaction, which does not reach the cortex trans-thalamically.

Thalamic relays differ with respect to whether their driver inputs arrive from the cortex (higher order $\mathrm{HO}$ or associational relays such as the pulvinar nucleus) or from subcortical sources (first order nuclei FO such as the LGN) (see Figure 1). Thus, FO relays transmit copies of motor commands issued by subcortical inputs to the thalamus, while the HO thalamic relays transmit copies of motor commands issued by cortical layer VB neurons. Another way to think about this is that the FO nuclei transmit information that has not been processed by a cortical area yet while the HO relays transmit information that has been processed by at least one cortical area.

All cortical areas, regardless of their classification as sensory, associational or motor, contain layer VB neurons with branching axons that issue motor commands to lower motor centers in the CNS and provide driver inputs to thalamic relays, which in turn relay this information to other cortical areas. We discussed one example of this earlier, namely, that of the primary visual cortex, which contains layer VB neurons that branch and simultaneously innervate the thalamus and lower motor centers. Therefore, one way to describe cortico-thalamo-cortical loops is that they are a substrate for an ongoing elaboration of copies of motor commands issued at all levels of the sensorimotor hierarchy.

These findings together challenge the sensory versus motor dichotomy within the thalamus and the cortex. Qualitatively speaking, all of the main informationcarrying inputs to the thalamus transmit information that is both sensory and motor. This means that no purely sensory information reaches the cortex trans-thalamically. The world of experience is an active representation. Our experience of the world emerges as motor commands are continually issued, copied, and then relayed transthalamically thereby creating both perceptual experience and behavior simultaneously, which are inextricably linked. The same neurons that issue motor commands to lower motor centers in the CNS branch and innervate tha- lamic relays that then provide their target cortical areas with their most direct access to the information about the body and the world. The mind perceiving the world corresponds to the brain detecting the copies of motor commands that it issues to the body.

The brain is therefore not merely an informationprocessing but also an information-generating structure (6). What it generates, among other things, is the human experience, which is, therefore, a model. Importantly, a hallmark of all models is that they have pre-conditions and purposes not contained within them. As an analogy, let us consider maps. What enables a map to function as such is not contained within it. It, by itself, does not contain its relation to a larger reality. Something else, not contained within a map, needs to decide how the map is related to what it is depicting and how to use it. For this reason, maps and other models usually function within the framework of something else (such as another more comprehensive model), which in turn provides the necessary pre-conditions and purposes.

Thus, because human experience of reality is necessarily a model, it is not an end in itself. As with other models and representations, its pre-conditions and purposes are not contained within. For example, sociologists argue that it is not possible to understand our sense of reality without appreciating how societal forces shape an individual's sense of what is real. Similarly, psychoanalysts hold that our sense of reality emerges from unconscious forces, and that conscious experiences are in the service of those forces. To use yet another example, dreams are also models whose preconditions lie within a larger framework provided by a more comprehensive awareness during wakefulness.

Our brains and our experience that it generates have evolved in order to allow our species to survive and to ultimately propagate our genetic material. Evolution, however, is itself a model, which is embedded in a more basic model provided by genetics and molecular biology. Molecular biology is in turn embedded into chemistry and chemistry into physics. Thus, to account for the human experience we need increasingly basic models, which science has been remarkably successful in providing. However, before we explore this further, let us investigate an instance of when human experience as a model malfunctions.

\section{The thalamus and schizophrenia}

Having described the basic thalamocortical neuroanatomy, and how it generates an individual's perceptual experience, let us now explore an important clinical correlate, which underscores the basic argument that the 
Figure 1. Comparison Between the Traditional View of Thalamocortical Functioning (A) and an Alternative View Proposed by Murray Sherman and Ray Guillery (B) (Adapted Adapted from (5))

A

Conventional View

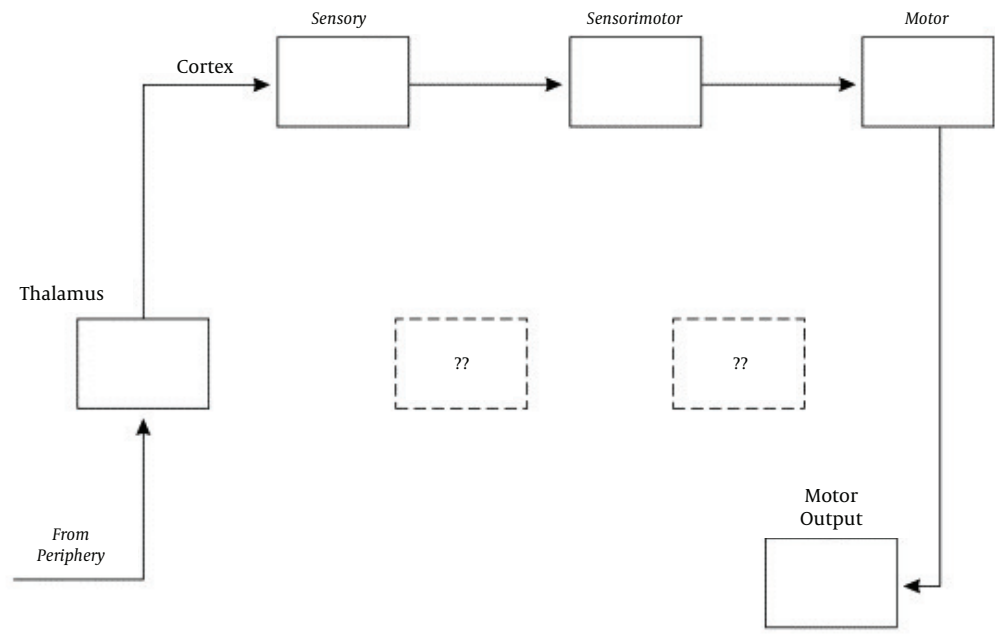

B Alternative View

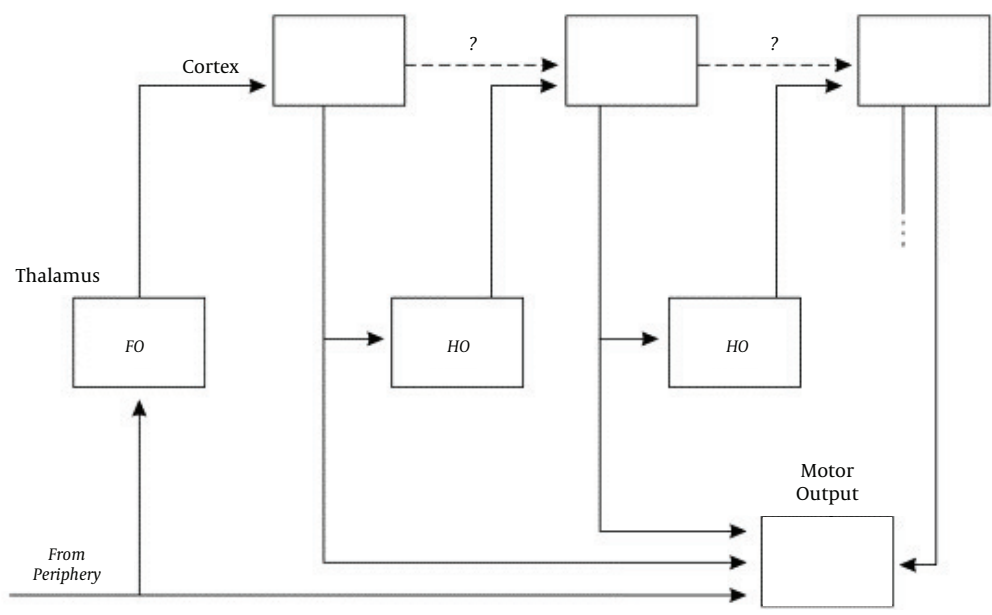

Interactions between the thalamus and the cortex correspond to how our mind experiences reality. According to the conventional view of thalamocortical functioning, the thalamus serves as a relay for sensory information from the bodily periphery to reach the cortex. The cortex then processes this information sequentially as it passes from sensory cortical areas to sensorimotor areas and then to motor areas. Motor cortical areas then issue commands to the body. In contrast, according to the alternative view based on the microarchitecture of thalamocortical networks, the main information-carrying inputs that reach the thalamus and then the cortex are actually copies of motor commands issued to lower motor centers in the central nervous system. Such information, that reaches the cortex via the first order thalamic nuclei (FO), has not been processed by any cortical region yet but nonetheless contains copies of motor commands already issued as this information is travelling to the thalamus. Higher order thalamic nuclei (HO) relay copies of motor commands issued to lower motor centers by cortical areas at all levels of sensory and motor cortical hierarchies. Because every cortical area issues motor commands, as well as copies of those commands to the thalamus, there is no qualitative division between sensory and motor processing in the cortex.

human experience is a model whose purpose is to enable human functioning and survival.

In schizophrenia, which is associated with profound perceptual disturbances and cognitive symptoms, there is converging evidence that trans-thalamic cortico-cortical interactions are impaired (7). Namely, this illness is associ- ated with thalamic abnormalities that affect the HO thalamic relays, which are tasked with relaying information between cortical areas. In an interesting study that combined functional neuroimaging and transcranial magnetic stimulation (TMS), Guller et al. (2012) found that TMS stimulation of the precentral gyrus in individuals with schizophre- 
nia resulted in reduced thalamic activation, which was correlated with the severity of positive symptoms in those individuals (8). They also reported reduced secondary cortical activation, which was statistically related to the reduced thalamic activation.

The converging evidence that schizophrenia involves reduced ability of the thalamus to support trans-thalamic cortico-cortical communication fits well with findings that the illness also involves failure of corollary discharge mechanisms. This term denotes processes whereby copies of motor commands are used to adjust sensory responsiveness in accordance with ongoing motor outputs. Failure of such processes may lead to perceptual abnormalities whereby sensory consequences of own motor outputs are misinterpreted as externally imposed, which in turn underlies positive symptoms such as auditory hallucinations and passivity experiences.

\section{Models and the Unmodelable}

As we have seen, human experience of reality is a model that as such requires external pre-conditions and purposes. Namely, models or representations are never self-sufficient; they always require something external to allow them to function as representations. As we have seen, this is usually provided by more basic and comprehensive models.

To avoid infinite regress by proposing ever more basic models to accommodate reality as we experience it, let us propose a model of all models, something like a theory of everything that many renowned physicists are currently working on. This model of all models, like all other models, still, however, requires an outside precondition to function as such. However, this cannot be yet another model because, by definition, it encompasses all models. Therefore, its precondition cannot be something that is modelable. It thus follows that the ultimate precondition for reality is something that is by definition unknowable.

The idea that what makes things intelligible is ultimately itself unknowable is reminiscent of Goedel's proof in mathematics. Kurt Goedel (1906 - 1978) was an Austrian born American mathematician and logician whose work in number theory demonstrated that there are true statements that cannot be formally proven. When this is attempted, inconsistencies emerge. In other words, the truth is not subordinate to provability (see Goedel, Escher, Bach by Douglas Hofstadter, 1979). What ultimately determines whether some propositions are true or not cannot itself be formalized and independently known.

What does it mean for something to be unknowable? As we have seen, one feature of all representations is that they have outside requirements that allow them to function as representations. Something that is by definition unknowable and cannot be represented is then also something that is fully self-sufficient. It, in contrast to things that are modelable, does not require any outside preconditions or purposes to be what it is. Thus, unknowability and self-determination are two related concepts, two sides of the same coin.

What does it mean for something to be selfdetermined? As argued by Baruch Spinoza, a 17th century philosopher, in his work Ethics, something that is by definition self-determined is also something that is boundless because it cannot be constrained by anything else. Because it is boundless, it is also infinite, all-encompassing and unique. In other words, it transcends everything else that exists. In Spinoza's terms, this is God.

\section{Summary: from Neuroscience to God}

In this commentary, we have attempted a remarkable journey. We have seen that from the perspective of neuroanatomy the information that reaches the cortex via the thalamus and that constitutes our conscious experience always contains copies of motor instructions issued to the body. Thus, we do not experience things as they are in themselves. Our experience is necessarily always a model. One feature of models and other representations such as maps is that they have pre-conditions and purposes not contained within them. For example, they can be embedded in another model, which then provides those outside requirements. However, then, this model is in the same position. It too has external requirements.

To ultimately explain how anything can be knowable, it is helpful to imagine a set that contains all models or a model of all models. The existence of something like this is being taken seriously by a number of contemporary physicist who are attempting to develop a theory of everything. However, even when and if we have such a theory, a model of all models, it will still be incomplete because like all models, it cannot be an end in itself. It will still have external pre-conditions and purposes. However, because this model of all models already contains everything that can be represented and known, its external requirement cannot be something that is itself knowable. Being unknowable by definition implies self-determinism and self-determinism implies boundlessness. Boundlessness in turn implies transcendence, infinity and uniqueness. Hereby we see that contemporary neuroscience is compatible with the belief in one God. 


\section{References}

1. Llinas RR. I of vortex: From neurons to self. Cambridge: The MIT Press; 2002.

2. Alkire MT, Miller J. General anesthesia and the neural correlates of consciousness. Prog Brain Res. 2005;150:229-44. doi: 10.1016/S00796123(05)50017-7. [PubMed: 16186027].

3. Viticchi G, Falsetti L, Fiori C, Jorio G, Plutino A, Buratti L, et al. Acute Occlusion of the Percheron Artery during Pregnancy: A Case Report and a Review of the Literature. J Stroke Cerebrovasc Dis. 2016;25(3):572-7. doi: 10.1016/j.jstrokecerebrovasdis.2015.11.011. [PubMed: 26698643].

4. Sherman SM, Guillery R. Functional connections of cortical areas: A new view from the thalamus. Cambridge: MIT Press; 2013.
5. Sherman SM, Guillery R. Thalamic Relays and Cortical Functioning. 149. Brain Res: Prog; 2005. pp. 107-26.

6. Buzsaki G. Rhythms of the Brain. New York: Oxford University Press; 2006.

7. Vukadinovic Z. Sleep abnormalities in schizophrenia may suggest impaired trans-thalamic cortico-cortical communication: towards a dynamic model of the illness. Eur J Neurosci. 2011;34(7):1031-9. doi: 10.1111/j.1460-9568.2011.07822.x. [PubMed: 21895800].

8. Guller Y, Ferrarelli F, Shackman AJ, Sarasso S, Peterson MJ, Langheim FJ, et al. Probing thalamic integrity in schizophrenia using concurrent transcranial magnetic stimulation and functional magnetic resonance imaging. Arch Gen Psychiatry. 2012;69(7):662-71. doi: 10.1001/archgenpsychiatry.2012.23. [PubMed: 22393203]. 